ded on biochemical tests, but this difference was minimized when identification depended upon a technique such as Lancefield grouping. Performance in antibiotic sensitivity testing did not differ significantly with the two specimen types.

Urinary cytology in the assessment of progress after renal transplantation

MARGARET BISSON, P. BISSON, D. SPRATT, AND SYLVIA DAVIES (Renal Unit, Whipton Hospital and Area Department of Pathology, Exeter) Cytological examination of the urine has provided immediate indication of early rejection after renal transplantation. The appearance of activated lymphocytes and immunoblasts in the urinary deposit correlates well with other parameters of rejection, such as reduced renal function and increased peripheral blood lymphocyte count. Other abnormalities, including tubular necrosis and infection, can be identified and differentiated from those of rejection. Results can be reported within an hour after receipt of a fresh sample of urine.

\section{Hyphomycetes in the hands of laymen}

D. O. HAINES (Royal Naval Hospital, Haslar, Hampshire) Chance consultation in 1973 led the author into an otherwise foreign territory when asked to provide services to a (non-microbiological) products assessment laboratory. The service required was to prepare and standardize fungal spore suspensions during comparative testing of US and British standards for resistance to mould growth of electronics equipment and assemblies.

This, because of the now recognized infectivity, especially in certain chronically ill or iatrogenically induced sub-immune workers, of some of the organisms used, especially species of non-pathogenic aspergillus, has brought about a re-examination of the principles, scientific validity, and technical performance of these tests.

Examination of mortality statistics and PHLS reports has not enabled any direct link between cases of Aspergillus infection and work in factories employing mould growth resistance tests to be made. The author would be grateful for reports from morbid anatomists and microbiologists coming across patients suffering from Aspergillus infection or allergy whose occupation may have been the source. The address for any such reports, which will receive 'medical and commercial confidential' handling is: $\mathrm{Dr} D$. O.
Haines, Health and Hygiene Laboratories, Health and Safety Executive, 403405 Edgware Road, Cricklewood, London NW2 6LN.

Leucocyte ascorbic acid in abnormal leucocyte states

G. M. G. BARTON AND O. S. ROATH (Department of Pathology, General Infirmary, Salisbury, and Department of Haematology, Southampton University) The leucocyte ascorbic acid (LAA) content is the most commonly used method of examining body vitamin $\mathrm{C}$ status, but no corrections or interpretations are made relating to the type or maturity of the leucocytes or whether a recognizable leucocyte disorder is present. Disorders with a high preponderance of one type of leucocyte were therefore investigated, noting that abnormal or immature cells might be present. The LAA was estimated in cases of infectious mononucleosis, reactive leucocytosis, and various types of leukaemia. Note was made of patients on cytotoxic drugs. Results show that the LAA content in most of the leukaemias, acute and chronic, was abnormally low. In infectious mononucleosis, some $50 \%$ were low. In patients with leucocytosis there was a wide scatter of levels. About half of the patients on cytotoxic drugs showed low levels. This might reflect their underlying condition. A significant depression of LAA values was found in pregnant patients. In none of these subjects was there any evidence of overt scorbutism or deficiency of vitamin $\mathrm{C}$ in the diet. It is therefore concluded from these studies that LAA resides largely in normal mature polymorphs, and that its assay in abnormal leucocyte states may be misleading as an index of body vitamin C status.

Value of microbiological examination at necropsy on the newborn

ROSALINDE HURLEY AND J. PRYSE-DAVIES (Institute of Obstetrics and Gynaecology, Queen Charlotte's Hospital for Women, London) Microbiological examination was made on material taken at necropsy on 585 of $613(95.4 \%)$ neonates over a seven-year period. The specimens examined included cerebrospinal fluid, heart blood, and bronchial swabs. Bacteriological studies were made routinely, and virological studies where indicated by the necropsy findings. The microbiological findings, particularly those relating to neonatal meningitis or septicaemia, will be described briefly, and the value of thet investigations as a routine adjunct to necropsy will be illustrated.

Grade of histological differentiation, gluts mate dehydrogenase activity and alphafeto protein production in human hepatocellula: carcinoma

P. P. ANTHONY (Bland-Sutton Institute of Pathology, Middlesex Hospital, London, C. L. VOGEL AND R. I. GLAZER (Emorף University, Atlanta, Georgia, USA) and K. R. MCINTIRE (National Cancer Institut Bethesda, Maryland, USA) Activity of glutamate dehydrogenase (GDH) in the liver and serum levels of alphafetoprotein (AFP) were correlated with the degree of histological differentiation in cases of human hepatocellular carcinoma to see of a metabolic behaviour pattern, similar to that seen in experimental animal tumour引, could be detected.

GHD activity was measured spectr@ photometrically in tumour tissue as well

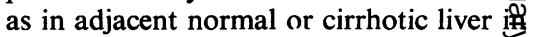
19 patients. AFP levels were determined. in these as well as in a further 163 patient9 by qualitative immunodiffusion and quẹp titative radioimmunoassay.

No differences in GDH activity detected between normal or cirrhotic Five tissue. In hepatocellular carcinoma tissu民, enzyme activity was significantly reduceg and this decrease was proportionate greater in poorly differentiated tumours In this latter group qualitative immunos diffusion for AFP was more frequently positive and quantitative radioimmunoas say showed higher serum levels. Concukrent estimations of serum protocollagen proline hydroxylase, glutamic-oxalacet!f transaminase, and alkaline phosphatase. showed elevated levels in most patients irrespective of the grade of tumour.

These data suggest that patterns of metabolic behaviour exist in huma hepatocellular carcinoma which can bo related to the degree of differentiation an growth rate of the tumour.

Fatal post-traumatic vertebro-basilar iscis aemia

R. A. GOODBODY (Department of Neuro pathology, Southampton University Generg Hospital) Hutchinson and Yates (196 reported spasm and thrombosis of art extracranial carotid artery as being responsible for fatal cerebral infarctio Animal experiments have suggested tha simple spasm of these arteries may tof 